\title{
ZIRCÔNIA NA ODONTOLOGIA: MINI-REVISÃO
}

\author{
Zirconia in Dentistry: mini-review
}

DSimone Kreve ${ }^{a}$, IDAndréa Cândido dos Reis ${ }^{\mathrm{b}}$

\section{RESUMO}

Objetivo: O objetivo deste estudo é fornecer ao cirurgião-dentista conhecimento sobre aspectos gerais da zircônia e abordar a introdução das diferentes gerações. Materiais e métodos: Foi realizada uma pesquisa eletrônica da literatura atual em inglês, incluindo artigos científicos publicados até 2019. Resultados: De acordo com os artigos selecionados, diferenças importantes foram encontradas em relação as novas composições da zircônia, sua microestrutura, diferenças no conteúdo da fase cúbica, e de ítria na fase tetragonal, levando a diferentes propriedades ópticas, mecânicas e de resistência ao envelhecimento. Conclusão: A zircônia é o material mais resistente disponível entre as cerâmicas, com diferenças mecânicas e ópticas entre os materiais disponíveis. A introdução das coroas monolíticas resolveu o problema de lascamento da porcelana de cobertura, mas foram necessárias modificações estruturais para fornecer adequada translucidez.

Palavras-chave: Cerâmica. Zircônio. Próteses e implantes.

\begin{abstract}
Purpose: This study aims to provide knowledge to clinicians about general aspects of zirconia and to approach the introduction of different zirconia generations. Methods: An electronic search of the English current literature was conducted including scientific articles published up to 2019. Results: According to the selected articles, important differences were found in terms of the novel compositions of zirconia, microstructures among the materials, differences in cubic phase content and yttria in the tetragonal phase, leading to different optical, mechanical and aging resistance properties. Conclusion: Zirconia is the most resistant material available among ceramics with mechanical and optical differences on available materials. The introduction of the monolithic crowns solving the veneering delamination but structural modifications were necessary to provide adequate translucency.
\end{abstract}

Keywords: Ceramics. Zirconium. Prostheses and implants.

\footnotetext{
aDoutoranda em Reabilitação Oral, Departamento de Materiais Dentários e Prótese, Faculdade de Odontologia de Ribeirão Preto, Universidade de São Paulo FORP/USP, Ribeirão Preto, SP, Brasil.

bDocente do Departamento de Materiais Dentários e Prótese, Faculdade de Odontologia de Ribeirão Preto, Universidade de São Paulo-FORP/ USP, Ribeirão Preto, SP, Brasil.
}

Autora de correspondência: Andréa Cândido dos Reis - E-mail: andreare73@yahoo.com.br

Data de envio: 07/04/2020 | Data de aceite: 01/06/2020 


\section{INTRODUÇÃO}

A zircônia é o material mais resistente dentre as cerâmicas odontológicas disponíveis, com crescente uso na prática clínica e indicada para tratamentos desde uma prótese fixa unitária a estruturas de próteses fixa sobre implantes e componentes protéticos.

A zircônia ou dióxido de zircônio $\left(\mathrm{ZrO}_{2}\right)$ é um óxido cristalino do zircônio que está presente na natureza em conjunto com óxidos, e após sua purificação é disponibilizada na forma de um pó branco e cristalino ${ }^{1-3}$. Possui três formas cristalográficas: monoclinica $(M)$, cúbica (C) e tetragonal $(\mathrm{T})^{4,5}$. A fase cúbica é estável acima de $2.370^{\circ} \mathrm{C}$ e possui propriedades mecânicas moderadas, a fase tetragonal é estável entre $1.170^{\circ} \mathrm{C}$ e $2.370^{\circ} \mathrm{C}$ e possibilita propriedades mecânicas aprimoradas, enquanto a fase monoclínica é estável em temperaturas ambiente até $1.170^{\circ} \mathrm{C}$, e apresenta desempenho mecânico reduzido ${ }^{3,6}$. Sendo a zircônia pura monoclínica à temperatura ambiente, a mesma não pode ser utilizada para formar cerâmicas odontológicas devido ao fraco desempenho mecânico ${ }^{1,7}$ entretanto, ao mistura-la com outros óxidos, como $\mathrm{MgO}$, $\mathrm{CaO}$ ou $\mathrm{Y}_{2} \mathrm{O}_{3}$, é possível obter estabilidade molecular pela geração de um material multifásico à temperatura ambiente ${ }^{8}$. O sucesso desse material na medicina e odontologia se deve a transformação termomecânica induzida da fase tetragonal para a fase monoclínica ${ }^{2,8}$. Essa transformação provoca um aumento de volume gerando tensões compressivas superficiais impedindo a propagação de trincas como uma resistência adicional, mecanismo conhecido como tenacificação $0^{4}$. Tal propriedade explica a alta resistência à fratura da zircônia ${ }^{8}$.

Como características a zircônia é durável, resistente ao desgaste e à corrosão, biocompatível, hipoalergênica, possui baixa toxicidade, é quimicamente inerte, apresenta menor adesão de bactérias se comparada ao titânio ${ }^{1,4,7,9,10}$ e ainda, possui propriedades mecânicas semelhantes ao aço ${ }^{8,11}$.

Diversos materiais de zircônia foram constituídos, dentre eles, policristais de zircônia tetragonal estabilizada com $3 \mathrm{~mol} \%, 4 \mathrm{~mol} \%$, e $5 \mathrm{~mol} \%$ de ítrio $^{1,12-14}$ e diferentes quantidades de alumina $\left(\mathrm{Al}_{2} \mathrm{O}_{3}\right) \cdot{ }^{15}$ zircônia parcialmente estabilizada com magnésio (Mg-PSZ) e por cério ${ }^{1}$ sendo que as diferenças estão nas propriedades ópticas e mecânicas.

A introdução da zircônia na odontologia se deu início dos anos $90^{5}$ para ser utilizada como infraestrutura para próteses fixas ${ }^{11} \mathrm{e}$ com a conformação de blocos para usinagem pela tecnologia CAD/CAM, a qual permite que as próteses sejam produzidas no consultório odontológico, em um laboratório ou centro de fresagem ${ }^{16}$. O CAD usa um software para definir a forma e as dimensões da restauração, enquanto o CAM proporciona que o modelo projetado seja fabricado ${ }^{17-19}$. O sistema CAD/CAM possui vantagens de reprodutibilidade da prótese, redução nos custos laboratoriais, facilidade no polimento, resistência à fratura por possuir padronização de fábrica, além de ser obtido com rapidez e precisão ${ }^{4,5,11,12,17}$, tornou-se um componente importante nos tratamentos protéticos contemporâneos sem metal ${ }^{5}$.

Considerando que o sucesso clínico está relacionado com a correta aplicação ${ }^{12}$, o objetivo desse estudo é simplificar o entendimento descrevendo os aspectos gerais da zircônia e as diferentes gerações desse material.

\section{MATERIAIS E MÉTODOS}

Para a execução deste trabalho, do tipo revisão de literatura, foram utilizadas publicações livros e artigos científicos - nas bases de dados eletrônicas: PubMed, Portal de Periódicos CAPES/MEC, Elsevier além de buscas manuais nas referências dos artigos selecionados. Foram consultados materiais escritos em inglês e de periódicos indexados na base Journal Citation Reports (JCR). As palavras-chaves utilizadas foram: zircônia, reabilitação protética, biomaterial cerâmico. 


\section{ASPECTOS GERAIS DA ZIRCÔNIA}

A zircônia apresenta algumas propriedades mecânicas com valores aumentados, como a tenacidade à fratura e a resistência à flexã $0^{13}$, se comparados a outros materiais cerâmicos como dissilicato de lítio e cerâmica feldspática ${ }^{20}$.

A resistência à flexão da zircônia é comparada ao aço $\mathrm{O}^{11}$, e tal propriedade pode ter sua importância exemplificada em uma prótese de três elementos, onde, sob tensões de flexão no pôntico, a zircônia (900-1200 MPa) é praticamente 3 vezes mais resistente que o dissilicato de lítio (262-306 MPa). Devido a zircônia possuir módulo de elasticidade elevado (210 GPa) a mesma é rígida e por isso indicada para confecção de estruturas para prótese sobre implantes ${ }^{12}$ copings para prótese fixa unitária ${ }^{5}$ e próteses parciais fixas posteriores (DPFs), inclusive com espessura diminuída $a^{4,11,12,14}$.

A cerâmica odontológica independentemente se feldspática ou reforçada, (p. exemplo por dissilicato de lítio, alumina, leucita) continua sendo um material frágil, com baixa tolerância a tensões de tração e cisalhamento, e suscetível à formação e propagação de trincas por ser friável e de baixa resistência ao impacto ${ }^{13}$. A tenacidade a fratura da zircônia, que é a capacidade da cerâmica resistir à fratura quando uma trinca esta presente, continua sendo baixa ${ }^{10,17}$, de 4 a 5 MPa.m, ${ }^{0.5}$ no entanto um pouco maior que do dissilicato de lítio que é de 3 MPa.m ${ }^{0.5}$ e da cerâmica feldspática que é $0,78 \mathrm{MPa} \cdot \mathrm{m}^{0.5}$, e possivelmente esse valor aumentado esta relacionado com a capacidade de tenacificação por transformação que será explicado mais adiante.

A zircônia $\left(\mathrm{ZrO}_{2}\right)$ apresenta três formas cristalográficas bem definidas, a monoclínica (estável até $1150^{\circ} \mathrm{C}$ ), e após essa temperatura se transforma em tetragonal (estável até $2370^{\circ} \mathrm{C}$ ) e a cúbica (até o ponto de fusão, a $\left.2680^{\circ} \mathrm{C}\right)^{4-6}$ sendo que a forma tetragonal oferece as melhores propriedades mecânicas ${ }^{5,13,16,21}$.

A transformação de fases na zircônia ocorre quando a cerâmica é submetida à tensão e/ou temperatura, principalmente na presença de água ${ }^{6,21}$. A transformação da estrutura cristalina de tetragonal para monoclínica $(\mathrm{t} \rightarrow \mathrm{m})$ é chamada de transformação martensítica e ocorre no resfriamento de $1300^{\circ} \mathrm{C}$ até a temperatura ambiente $e^{4,6,13,16}$.

A utilização do dióxido de zircônia puro não é interessante para confecção de próteses odontológicas, uma vez que, durante a transformação martensítica ocorre um aumento abrupto de volume, e como resultado podem ocorrer trincas, além de que na fase monoclínica a zircônia é muito frágil ${ }^{1,20}$.

A transformação martensítica pode ser evitada, alterando-se a composição da zircônia pela adição de estabilizadores como $\mathrm{Mg}$, Ca, Y e Ce, o que permite que a fase tetragonal de alta temperatura seja estabilizada a temperatura ambiente, ou seja, a fase que é obtida as custas de alta temperatura se mantem fixa mesmo em temperatura ambiente, na condição tetragonal ou na nova condição cúbico-tetragonal ${ }^{1,21,22}$. Dessa forma as tensões de transformação são evitadas e as propriedades mecânicas positivas como maior tenacidade à fratura e resistência à flexão da fase tetragonal são preservadas ${ }^{6,10,21}$.

A quantidade de óxidos, também chamados de estabilizadores ou dopantes , $^{4,13,21,23}$, é incorporada em pequenas concentrações pré-estabelecidas para determinar a estabilidade de fase, a transformabilidade e as propriedades mecânicas ${ }^{22}$. Quando adicionada em quantidades maiores (>12\%), uma fase cúbica totalmente estabilizada pode ser produzida, o que inviabiliza a transformação de fase tetragonal-monoclínica, resultando em pior desempenho ${ }^{24,25}$.

A ítria $\left(\mathrm{Y}_{2} \mathrm{O}_{3}\right)$ é um metal prateado, sólido e brilhante e até o momento da realização deste estudo, ela provou ser o óxido mais eficaz na combinação de alta resistência e tenacidade ${ }^{4}$, e possui propriedades mecânicas superiores devido ao seu tamanho de grão relativamente fino acoplado à transformação de fase tetragonal em monoclínica ${ }^{22}$. 
Outro importante aspecto da zircônia é seu mecanismo único para impedir o avanço de trinca, que foi descoberto na década de $1970^{5}$. Tensões de compressão são geradas nas pontas das trincas, que parecem fechá-las e impedir a propagação ${ }^{22}$. Esta transformação pode ser induzida por fatores termomecânicos ${ }^{5}$.

Após adição de ítria, a zircônia tetragonal é estável em temperatura ambiente, porém, sob tensão, como por exemplo ao redor de trincas, esta fase pode sofrer alteração para a fase monoclínica, a qual naturalmente produz um aumento de volume $e^{22,24}$. Esse aumento de volume causa compressão no local da trinca evitando assim, seu crescimento e propagação ${ }^{8}$. Este mecanismo, conhecido como “tenacificação por transformação", é o principal responsável pelas superiores propriedades mecânicas da zircônia ${ }^{5,13}$.

A estabilização da fase tetragonal se origina de uma combinação de efeitos da energia superficial, tamanho da matriz (tamanho dos grãos de zircônia) e adições de óxido estabilizador $^{7}$. Falhas microestruturais podem ser minimizadas pela utilização de partículas de $\mathrm{ZrO}_{2}$ de tamanho nanométrico, pois as mesmas não aglomeram e permanecem distribuídas de maneira homogênea, assim é possível reter uma quantidade maior de grãos de zircônia tetragonal na matriz de alumina e isso contribui para o mecanismo de transformação?.

\section{GERAÇÕES DE ZIRCÔNIA}

O contínuo desenvolvimento da zircônia mostra os esforços em melhorar a translucidez $^{22}$ sem comprometer indevidamente a integridade mecânica ${ }^{4}$ permitindo uso clínico com longevidade. A seguir estão descritas as diferenças na constituição e a indicação das quatro gerações de zircônia disponíveis.

\section{1. ${ }^{\mathrm{a}}$ Geração}

É a mais conhecida e constituída pela zircônia tetragonal parcialmente estabilizada por ítria (3Y-TZP). Possui características similares as cerâmicas feldspáticas e vítreas como estabilidade química, baixo potencial de corrosão, de desgaste, biocompatibilidade, dentre outras, integração com tecidos moles $^{18}$, e além disso, apresenta resistência mecânica elevada devido ao mecanismo de "tenacificação por transformação" ${ }_{5,21,24}$. Possui na sua composição, $0,25 \%$ em peso de alumina $\left(\mathrm{Al}_{2} \mathrm{O}_{3}\right)^{4,5,13}$ e grãos grandes com numerosas estruturas cristalinas muito pequenas pelas quais a luz precisa passar ${ }^{1,12,20}$ o que torna a zircônia bastante opaca ${ }^{1,4,12,18}$. Como desvantagem a zircônia de primeira geração apresenta falhas por lascamento ${ }^{12,14,18,24}$ pois necessita de uma cerâmica de revestimento para aumentar a translucidez superficial ${ }^{4} \mathrm{e}$ esta possui coeficiente de expansão térmica diferente da zircônia, gerando tensões residuais. O lascamento também pode surgir devido a taxas de resfriamento inadequadas e ciclos de queima durante a sinterização da porcelana de revestimento ${ }^{18}$.

Elas são indicadas principalmente para copings com recobrimento de porcelana tanto para dentes anteriores quanto posteriores, para confecção de infraestruturas, para o mascaramento de substratos escurecidos, como uma alternativa aos implantes e pilares de titânio ${ }^{4,12}$.

\section{2. ${ }^{a}$ Geração}

Por volta de 2013 foi apresentada uma versão aprimorada da zircônia e teve como principal diferença uma redução na quantidade e no tamanho dos grãos de alumina ${ }^{4,5,26}$, bem como eliminação da porosidade por sinterização a uma temperatura mais alta ${ }^{4,13,22}$. Essa alteração provocou uma melhora na passagem de luz e consequentemente na translucidez, 
entretanto, ainda não foi suficiente para que o material pudesse ser empregado em próteses monolíticas ${ }^{4}$. A alumina ajuda na sinterização, mas reduz a translucidez pois os grãos são menores e segregados próximos dos grãos zircônia ${ }^{15}$, no entanto os grãos maiores da primeira geração não possibilitavam a difusão da luz ${ }^{15}$. Devido à manutenção das ótimas propriedades mecânicas, esse tipo de zircônia é predominantemente usada como material de estrutura para próteses fixas unitárias e múltiplas ${ }^{12}$.

\section{3. ${ }^{a}$ Geração}

A zircônia de terceira geração foi introduzida no "The International Dental Show" em $2015^{1} \mathrm{com}$ intuito de incluir translucidez $z^{4}$. Além da fase tetragonal, foi adicionada uma proporção de fase cúbica de até $53 \%^{1}$ e alterada a quantidade de ítria para aproximadamente $5 \%$ em mol (5Y-PSZ)4. Os cristais cúbicos têm um volume maior em comparação aos tetragonais, o que faz com que a luz se espalhe menos fortemente nos limites dos grãos e nos poros residuais, tornando o material mais translúcido ${ }^{1}$. Além disso, as estruturas cristalinas cúbicas são mais isotrópicas que as estruturas tetragonais, e a luz incidente é emitida de maneira mais uniforme em todas as direções espaciais ${ }^{1,24}$. Entretanto, o aumento da translucidez levou a uma menor resistência à flexão $0^{20,25,27}$, e a fratura ${ }^{20}$, a qual não sofre transformação induzida por estresse $e^{4}$. Estas zircônias são indicadas para facetas cerâmicas e coroas monolíticas, coroas e próteses fixas anteriores, com até três elementos sendo um pôntico entre duas coroas na região de pré-molar ${ }^{4,12}$.

\section{4. ${ }^{\mathrm{a}}$ Geração}

Foi introduzida em 2017 e teve o teor de ítria reduzido para $4 \mathrm{~mol} \%$ a fim de aprimorar as propriedades mecânicas com uma redução combinada em suas propriedades ópticas ${ }^{12,15}$. Também estão disponíveis blocos com graduação de cor e de translucidez, visando alcançar propriedades (mecânicas, químicas e/ou ópticas) otimizadas. Dependendo do fabricante, a zircônia de quarta geração é indicada para próteses dentais fixas de vários elementos ${ }^{12}$.

Ao mesmo tempo em que as zircônias de $4 .{ }^{a}$ geração foram disponibilizadas, surgiram blocos com multicamadas ou sombreado com gradiente imitando a cor dos dentes naturais ${ }^{4,12,17}$. Além disso, foram introduzidos blocos que combinam diferentes gerações de zircônia para obtenção das melhores propriedades mecânicas e ópticas possíveis ${ }^{12}$. Esses blocos consistem em um corpo de zircônia de quarta geração (4Y-TZP) com melhores propriedades mecânicas e um material de terceira geração (5Y-TZP) com maior translucidez na área incisal, para permitir propriedades ópticas aprimoradas. E ainda, é possível encontrar blocos de zircônia com a integração de partículas fluorescentes ${ }^{12}$.

A busca por melhores resultados estéticos propicia que novos materiais e técnicas sejam devolvidos em substituição aos metais, e nesse contexto as diferentes gerações de zircônia foram introduzidas para equalizar estética com propriedades mecânicas.

\section{TAXAS DE FALHA DE TRATAMENTOS COM ZIRCÔNIA}

O desenvolvimento de cerâmicas de alta resistência levou ao aumento no uso da zircônia estabilizada com ítria (3Y-TZP) em aplicações odontológicas, entretanto as possíveis falhas são fatores de preocupação.

Umas das falhas é o lascamento da porcelana de cobertura aplicada sobre a zircônia parcialmente estabilizada por ítria (YPSZ). A YPSZ é uma cerâmica de alta resistência que 
se tornou amplamente utilizada para fabricação de copings devido à sua excepcional tenacidade. Entretanto, essa cerâmica não possui estética suficiente e necessita da aplicação de uma porcelana de cobertura, além de que ela possui dureza suficiente para induzir o desgaste dos dentes antagonistas durante a oclusão ${ }^{28}$.

A falha de lascamento geralmente ocorre próxima a interface coping/cerâmica de cobertura, e o acúmulo de tensões residuais nessa interface foi proposto como uma possível explicação ${ }^{28}$. Tensões são induzidas pelo coeficiente de incompatibilidade de expansão térmica entre o YPSZ e a porcelana de cobertura ${ }^{28}$. O lascamento também foi atribuído ao design da estrutura, a baixa resistência da cerâmica de revestimento ou ao resfriamento insuficiente durante o processo de revestimento ${ }^{14}$. Lunt et al. ${ }^{28}$ observaram que a presença de YPSZ na forma monoclínica nos primeiros micrômeros da interface, a existência de transformação tetragonal para monoclínica e o creep da porcelana próxima da interface são fatores consideráveis para induzir a falha por lascamento. Observaram ainda que a aplicação de cerâmica de cobertura formando facetas espessas resulta em um aumento das taxas de falhas nas próteses, pois a força para equilibrar o coeficiente de expansão térmica gera grandes tensões de tração na interface YPSZ-porcelana de cobertura. Weigl et al. ${ }^{14}$ também observaram desempenho significativamente diferente dependendo da espessura.

Outro fator que pode levar a um aumento ou diminuição da integridade da prótese é o tratamento térmico que a peça recebe para aderir cada camada da cerâmica de cobertura. Durante a fabricação, a pasta de porcelana de cobertura é aplicada ao coping YPSZ já sinterizado e que se encontra à temperatura ambiente. Após aplicação da camada de cerâmica, o coping é aquecido em um forno a vácuo a $750^{\circ} \mathrm{C}$. Durante o resfriamento, o coeficiente de expansão térmica incompatível entre o YPSZ e a porcelana causa uma tensão com pico na interface ${ }^{28}$.

A fim de aumentar a confiabilidade das próteses, foi proposto um tratamento superficial abrasivo na superfície YPSZ ${ }^{28}$. O jateamento induz mudança de fase tetragonal para monoclínica em escala micrométrica, assim essa abordagem reduz a extensão da transformação adicional durante o ciclo de sinterização, minimizando os efeitos de acúmulo de tensão residual e de creep na porcelana de cobertura ${ }^{28}$. Entretanto Kelch et al. ${ }^{5}$, mostrou que um aumento no tamanho das partículas e na pressão provocou aumento no conteúdo da fase monoclínica, sendo que $105 \mu \mathrm{m}$ de pó de alumina teve a maior quantidade de fase transformada e promoveu alteração na resistência a flexão. Assim sendo, $30 \mu \mathrm{m}$ de pó de alumina foi mais indicado.

E ainda, quanto mais compatível for a expansão térmica, mais confiável será a prótese. Ao reduzir a incompatibilidade de deformação induzida durante o resfriamento, o acúmulo de tensão residual e os danos induzidos por creep na porcelana próxima da interface são minimizados.

Uma outra alternativa para melhorar a confiabilidade das próteses é criar desenhos alternativos para melhorar a durabilidade sem comprometer muito a estética, tanto para peças monolíticas quanto para híbridas ${ }^{18}$. De acordo com Flinn et al ${ }^{18}$ utilizar próteses monolíticas com contornos anatômicos ou próteses híbridas projetadas para contornos anatômicos e com espaço para cerâmica apenas nas áreas estéticas são opções viáveis.

Ainda no intuito de solucionar a falha de lascamento da cerâmica de cobertura, foi incentivada a utilização de próteses de zircônia monolítica. Entretanto a preocupação está na degradação hidrotérmica de baixa temperatura (LTD) que é um fenômeno de envelhecimento lento que inicia na superfície do material quando em contato com a água, e que induz uma alteração na estrutura cristalina metaestável da zircônia ${ }^{5,6,18,29}$.

A Y-TZP é um material cerâmico policristalino em um estado metaestável, e como mencionado anteriormente, o óxido de ítrio atua como um dopante para estabilizar a forma tetragonal cristalina à temperatura ambiente, sendo que essa forma tetragonal pode se 
transformar na forma monoclínica sob efeito de estresse ${ }^{5,29}$. Entretanto, essa transformação de fase também pode ocorrer com o passar do tempo com a penetração de água na estrutura cristalina ${ }^{6}$. O estresse mecânico, a temperatura, a umidade e a mastigação, características do ambiente oral, são condições ideais para o desenvolvimento do LTD, o que impacta o prognóstico das próteses dentárias causando diminuição nas propriedades mecânicas, risco de fratura ${ }^{24,29}$, além de alterações microestruturais através de alterações superficiais que resultam em microfissuras e rugosidades na superfície $e^{6}$. O LTD ocorre nas restaurações monolíticas de zircônia que não são cobertas por uma camada de porcelana capaz de impedir a penetração de água ${ }^{29}$. Alguns fatores afetam a resistência da cerâmica para o envelhecimento como o teor de óxido de ítrio, o tamanho de grão, a densidade, a presença de uma fase cúbica e tensões residuais ${ }^{26}$.

De acordo com Kelch et $a .^{5}$, a resistência à flexão é a propriedade mecânica mais influenciada pelo envelhecimento, e depende da extensão e da propagação em profundidade da transformação da fase $\mathrm{t}-\mathrm{m}^{26}$.

Um procedimento comum é a retificação ou ajuste realizado na superfície oclusal das próteses durante os ajustes em consultório para obter uma oclusão ideal. Foi observado que a retificação suave da superfície aumenta a resistência da cerâmica ao envelhecimento nas proximidades da superfície. No entanto, a taxa de transformação aumenta quando o processo de transformação for além de $2 \mu \mathrm{m}$ de profundidade. Hatanaka et al. ${ }^{21} \mathrm{e}$ Cattani-Lorente et al. ${ }^{26}$ mostraram que o polimento além de reduzir a rugosidade e as falhas na superfície, melhorou a resistência à flexão da zircônia parcialmente estabilizada, sem afetar a zircônia totalmente estabilizada.

\section{CONCLUSÕES}

A literatura analisada demonstra que a zircônia é um material de alta qualidade para a fabricação de restaurações com aplicações clínicas variadas, e, de acordo com seu crescente desenvolvimento, a mesma alcançou melhorias nas propriedades ópticas. A literatura também demonstra que o sucesso clínico a longo prazo está fortemente vinculado à correta seleção de acordo com a indicação de cada material, sendo imprescindível que estas restaurações proporcionem estabilidade a longo prazo e reduzida sensibilidade aos efeitos do envelhecimento e de fadiga, o que pode ser vantajoso em condições clínicas.

\section{CONFLITO DE INTERESSES}

Os autores declaram não haver conflito de interesses.

\section{REFERÊNCIAS}

1. Stawarczyk B, Keul C, Eichberger M, Figge D, Edelhoff D, Lümkemann N. Three generations of zirconia: from veneered to monolithic. Part I. Quintessence int. 2017;48(5):369-80.

2. Piconi C, Maccauro G. Zirconia as a ceramic biomaterial. Biomaterials. 1999;20(1):1-25.

3. Vagkopoulou T, Koutayas SO, Koidis P, Strub JR. Zirconia in dentistry: part 1. Discovering the nature of an upcoming bioceramic. Eur] Esthet Dent. 2009;4(2):130-51.

4. Zhang Y, Lawn BR. Novel zirconia materials in dentistry. J Dent Res. 2018;97(2):140-7.

5. Kelch M, Schulz ], Edelhoff D, Sener B, Stawarczyk, B. Impact of different pretreatments and aging procedures on the flexural strength and phase structure of zirconia ceramics. Dent Mater. 2019;35(10):1439-49. 
6. Kim HK, Kim SH. Effect of hydrothermal aging on the optical properties of precolored dental monolithic zirconia ceramics. J Prosthet Dent. 2019;121(4):676-82.

7. De Aza AH, Chevalier ], Fantozzi G, Schehl M, Torrecillas R. Crack growth resistance of alumina, zirconia and zirconia toughened alumina ceramics for joint prostheses. Biomaterials. 2002;23(3):937-45.

8. Manicone PF, Iommetti PR, Raffaelli L. An overview of zirconia ceramics: basic properties and clinical applications. ] Dent. 2007:35(11):819-26.

9. Scarano A, Piattelli M, Caputi S, Favero GA, Piattelli A. Bacterial adhesion on commercially pure titanium and zirconium oxide disks: an in vivo human study. ] Periodontol. 2004:75(2):292-6.

10. Anusavice K], Shen C, Rawls HR. Phillips science of dental materials. 12. ed. Rio de Janeiro: Elsevier; 2013.

11. Malkondu Ö, Tinastepe N, Akan E, Kazazoğlu E. An overview of monolithic zirconia in dentistry. Biotechnol Biotechnol Equip. 2016;30(4):644-52.

12. Güth JF, Stawarczyk B, Edelhoff D, Liebermann A. Zirconia and its novel compositions: what do clinicians need to know? Quintessence Int. 2019;50(7):512-20.

13. Shahmiri R, Standard OC, Hart JN, Sorrell CC. Optical properties of zirconia ceramics for esthetic dental restorations: a systematic review. J Prosthet Dent. 2018;119(1):36-46.

14. Weigl P, Sander A, Wu Y, Felber R, Lauer HC, Rosentritt M. In-vitro performance and fracture strength of thin monolithic zirconia crowns. J Adv Prosthodont. 2018;10(2):79-84.

15. Jansen JU, Lümkemann N, Letz I, Pfefferle R, Sener B, Stawarczyk B. Impact of high-speed sintering on translucency, phase content, grain sizes, and flexural strength of 3 Y-TZP and 4Y-TZP zirconia materials. ] Prosthet Dent. 2019;122(4):396-403.

16. Meirowitz A, Bitterman Y, Levy S, Mijiritsky E, Dolev E. An in vitro evaluation of marginal fit zirconia crowns fabricated by a CAD-CAM dental laboratory and a milling center. BMC oral health. 2019;19(1):1-6.

17. Silva LHD, Miranda RBDP, Favero SS, Lohbauer U, Cesar PF. Dental ceramics: a review of new materials and processing methods. Braz Oral Res. 2017:31(1):133-46.

18. Flinn BD, Raigrodski A], Mancl LA, Toivola R, Kuykendall T. Influence of aging on flexural strength of translucent zirconia for monolithic restorations. ] Prosthet Dent. 2017;117(2):303-9.

19. Sen N, Us Y. Mechanical and optical properties of monolithic CAD-CAM restorative materials. J Prosthet Dent. 2018;119(4):593-9.

20. Zadeh PN, Lümkemann N, Sener B, Eichberger M, Stawarczyk B. Flexural strength, fracture toughness and translucency of cubic/tetragonal zirconia materials. J Prosthet Dent. 2018;120(6):948-54.

21. Hatanaka GR, Polli GS, Adabo GL. The mechanical behavior of high-translucent monolithic zirconia after adjustment and finishing procedures and artificial aging. J Prosthet Dent. 2019;123(2):330-37.

22. Tong H, Tanaka CB, Kaiser MR, Zhang Y. Characterization of three commercial Y-TZP ceramics produced for their high translucency. Ceram Int. 2016;42(1):1077-85.

23. Shenoy A, Shenoy N. Dental ceramics: an update. J Conserv Dent. 2010;13(4):195-203.

24. Camposilvan E, Leone R, Gremillard L, Sorrentino R, Zarone F, Ferrari M, et al. Aging resistance, mechanical properties and translucency of different yttria-stabilized zirconia ceramics for monolithic dental crown applications. Dent Mater. 2018;34(6):879-90.

25. Mao L, Kaizer MR, Zhao M, Guo B, Song YF, Zhang Y. Graded ultra-translucent zirconia (5Y-PSZ) for strength and functionalities. J Dent Res. 2018;97(11):1222-8.

26. Cattani-Lorente M, Durual S, Amez-Droz M, Wiskott HW, Scherrer SS. Hydrothermal degradation of a 3Y-TZP translucent dental ceramic: a comparison of numerical predictions with experimental data after 2 years of aging. Dent Mater. 2016;32(3):394-402.

27. Sulaiman TA, Abdulmajeed AA, Donovan TE, Cooper LF, Walter R. Fracture rate of monolithic zirconia restorations up to 5 years: a dental laboratory survey. J Prosthet Dent. 2016;116(3):436-39.

28. Lunt A, Salvati E, Baimpas N, Dolbnya I, Neo TK, Korsunsky AM. Investigations into the interface failure of yttria partially stabilised zirconia porcelain dental prostheses through microscale residual stress and phase quantification. Dent mater. 2019;35(11):1576-93.

29. Koenig V, Wulfman CP, Derbanne MA, Dupont NM, Le GoffSO, Tang ML, etal. Aging of monolithic zirconia dental prostheses: protocol for a 5-year prospective clinical study using ex vivo analyses. Contemp Clin Trials Commun. 2016;(4):25-32. 